\title{
NUMERICAL FLUX-SPLITTING FOR A CLASS OF HYPERBOLIC SYSTEMS WITH UNILATERAL CONSTRAINT
}

\author{
Florent Berthelin ${ }^{1}$
}

\begin{abstract}
We study in this paper some numerical schemes for hyperbolic systems with unilateral constraint. In particular, we deal with the scalar case, the isentropic gas dynamics system and the fullgas dynamics system. We prove the convergence of the scheme to an entropy solution of the isentropic gas dynamics with unilateral constraint on the density and mass loss. We also study the non-trivial steady states of the system.
\end{abstract}

Mathematics Subject Classification. 35A35, 35L65, 35L85, 76N15, 76T10.

Received: November 9, 2002.

\section{INTRODUCTION AND MODELS}

In various physical situations which naturally appear in fluid mechanics (two-phase flows, Saint-Venant equations, pipeline,...), it is necessary to impose constraints on some quantities of the model, for example, the fact that one quantity stays bounded by a fixed value. In gas dynamics, a lot of situations ask the density (or the volume fraction) to satisfy such a property. Two formulations are proposed and studied in a theoretical point of view in [1] and [4]. The aim of this paper is to introduce numerical schemes for these hyperbolic systems with unilateral constraint and to prove some convergence results. The model of [4] for the overflow of a river, which involves the density $\rho$ and the velocity $u$ of the fluid, can be written as

$$
\left\{\begin{array}{l}
\partial_{t} \rho+\partial_{x}(\rho u)=Q, \\
\partial_{t}(\rho u)+\partial_{x}\left(\rho u^{2}+p(\rho)\right)=Q u,
\end{array}\right.
$$

with constraint and mass loss rate Lagrange multiplier

$$
0 \leq \rho \leq 1, \quad Q \leq 0,
$$

and extremality relation

$$
(1-\rho) Q=0
$$

\footnotetext{
Keywords and phrases. Numerical scheme, conservation laws with constraint, convergence of scheme, entropy scheme, gas dynamics.

${ }^{1}$ Laboratoire J.A. Dieudonné, UMR 6621 CNRS, Université de Nice Sophia-Antipolis, Parc Valrose, 06108 Nice Cedex 2, France. e-mail: Florent.Berthelin@unice.fr 
The term $Q$ represents the lost mass. Existence and weak stability of suitable weak solutions is obtained in [4] in the pressureless case $p(\rho)=0$ and for the $\gamma$-law $p(\rho)=\kappa \rho^{\gamma}$.

In the present article, we study a class of hyperbolic system with unilateral constraint including this example. They are written as

$$
\partial_{t} U+\partial_{x} F(U)=Q \frac{U}{U_{1}},
$$

where $U=\left(U_{1}, \cdots, U_{p}\right) \in \mathbb{R}^{p}, F: \mathbb{R}^{p} \rightarrow \mathbb{R}^{p}$, and by definition

$$
\frac{U}{U_{1}}=\left(1, \frac{U_{2}}{U_{1}}, \cdots, \frac{U_{N}}{U_{1}}\right),
$$

and with constraint and mass loss rate Lagrange multiplier

$$
0 \leq U_{1} \leq 1, \quad Q \leq 0
$$

and extremality relation

$$
\left(1-U_{1}\right) Q=0 .
$$

The term $Q$ is the lost of the quantity $U_{1}$ which have to stay lesser than 1 . The term $Q \frac{U}{U_{1}}$ is for conservation reasons, the lost of the quantity $U$. There is a theoretical problem of definition as soon as $U_{1}=0$. We will come back on it in Section 2. The system (1.1)-(1.3) is an example of such problem with $U=(\rho, \rho u)$ and $F(U)=\left(\rho u, \rho u^{2}+p(\rho)\right)$.

The aim of this paper is to give a theoretical background for such system and some numerical flux-splitting schemes compatible with this theory. The remainder of the paper is organized as follows. In Section 2 , we present the necessary theoretical weak formulation and some numerical schemes which are compatible with it. We also treat the scalar case $(p=1)$. In Section 3, we study the isentropic system of Euler. In particular, we establish the convergence of a flux-splitting scheme with constraint to an entropy solution to the problem with mass loss. In Section 4, we analyze the steady states which are connected to this system with a source term. Finally, in Section 5, we briefly present an other example of system in this class of model, that is the full system of gas dynamics with constraints.

We also refer to $[10,11,18]$ and $[20]$ for other theoretical problems with constraints.

\section{General Formulations}

In this section, we first present a theoretical weak formulation in order to take into account the extremality relation (1.6) with the use of entropy inequalities. Then, we propose a numerical scheme which is compatible with the theoretical formulation. It is based upon a splitting and a projection onto the constraint states. We prove that an entropy method for an evolution problem without constraint gives an entropy method associated to the entropy formulation with a constraint. Finally, we study the scalar case.

\subsection{Theoretical weak formulation}

We notice in [4] that we have to give a suitable sense to the products $Q u$ in (1.1) and $\rho Q$ in (1.3), because $Q$ is only a measure, and $\rho, u$ can be discontinuous. We provide the following entropy weak formulation of the problem, that involves what we call entropy weak products. The idea is to introduce a different velocity $v(t, x)$ for the lost matter, and to write the system

$$
\left\{\begin{array}{l}
\partial_{t} \rho+\partial_{x}(\rho u)=Q \\
\partial_{t}(\rho u)+\partial_{x}\left(\rho u^{2}+p(\rho)\right)=Q v
\end{array}\right.
$$


with as before

$$
0 \leq \rho \leq 1, \quad Q \leq 0 .
$$

We take $v \in L^{\infty}(Q)$, so that the product $Q v$ is well-defined as a measure. We need then to formulate in a weak sense that $Q v=Q u$, and that $Q \rho=Q$. In order to do so, we require the family of entropy weak product inequalities

$$
\partial_{t} \eta_{S}(\rho, u)+\partial_{x} G_{S}(\rho, u) \leq Q \eta_{S}^{\prime}(1, v) \cdot(1, v)
$$

for any convex entropy $\eta_{S}$ in a suitable family parametrized by a convex function $S$, where $G_{S}$ is its entropy flux, and $\eta_{S}^{\prime}$ is its derivative with respect to $(\rho, \rho u)$. Since $v$, by definition, is defined $Q$ a.e., the term on the right-hand side of (2.3) is well-defined. Indeed, (2.1)-(2.3) is a weak formulation of (1.1)-(1.3).

We extend this formulation to more general system in introducing the following entropy weak product formulation. We introduce $V(t, x)$ for the lost matter where $V=\left(1, V_{2}, \cdots, V_{p}\right) \in \mathbb{R}^{p}$ with $V \in L^{\infty}(Q)$, and write the system as

with

$$
\partial_{t} U+\partial_{x} F(U)=Q V
$$

$$
0 \leq U_{1} \leq 1, \quad Q \leq 0 .
$$

In particular, it overcomes the problem of definition for $U / U_{1}$ when $U_{1}=0$. In order to have in a weak sense

$$
Q U_{1}=Q, \quad \text { and } \quad Q V=Q \frac{U}{U_{1}},
$$

we require a solution $(U, V, Q)$ of $(2.4,2.5)$ to satisfy the entropy inequalities

$$
\partial_{t} \eta(U)+\partial_{x} G(U) \leq Q \eta^{\prime}\left(1, V_{2}, \cdots, V_{p}\right) \cdot\left(1, V_{2}, \cdots, V_{p}\right),
$$

for a sufficient family of entropies $(\eta, G)$ associated to the system (1.4), and $\eta^{\prime}$ is its derivative with respect to $U$.

For a system with a strong entropy, for which we can prove a priori the condition

$$
Q V=Q \frac{U}{U_{1}}
$$

it gives a weak formulation of the problem thanks to the result.

Proposition 2.1. For a system such that there exists an entropy $\eta$ of class $C^{2}$ and such that $\eta^{\prime \prime}$ is positive definite, then the formulation (2.4), (2.5), (2.7), with the condition (2.8), is a weak formulation for (1.4)-(1.6).

Proof. We assume having a sufficiently smooth solution to (2.4), (2.5), (2.7). Multiplying (2.4) by $\eta^{\prime}(U)$ and comparing with (2.7), we get

$$
Q\left[\eta^{\prime}\left(U_{1}, U_{2}, \cdots, U_{p}\right)-\eta^{\prime}\left(1, V_{2}, \cdots, V_{p}\right)\right] \cdot\left(1, V_{2}, \cdots, V_{p}\right) \leq 0 .
$$

We assume the condition (2.8), thus

$$
Q \eta^{\prime}\left(U_{1}, U_{2}, \cdots, U_{p}\right)=Q \eta^{\prime}\left(U_{1}, U_{1} V_{2}, \cdots, U_{1} V_{p}\right),
$$

which leads to

with

$$
Q\left[\left(U_{1}-1\right) \int_{0}^{1} \eta^{\prime \prime}\left(\vartheta_{t}\right) \cdot\left(1, V_{2}, \cdots, V_{p}\right)^{2} \mathrm{~d} t\right] \leq 0
$$

With the hypothesis on $\eta$ and (2.5), we conclude that $Q U_{1}=Q$. 
Remark 2.1. In the case where (2.8) is satisfy, only one entropy is necessary. For a problem in which the condition (2.8) is not obvious to verify, the entropy weak product can have a more complicated structure. For the isentropic gas dynamics, we notice in [4] that (2.7) for $S(x)=x^{2} / 2$ is enough to have a weak formulation for $Q u=Q v$ and $Q \rho=Q$. It is a open question to know how many entropies are required in the general case. In Section 5, an other example of such situation is studied.

\subsection{The flux-splitting scheme with constraint}

We present the numerical treatment we perform to study such hyperbolic problems. In particular, we propose a general method in which entropy schemes are still compatible with the taking into account of constraints.

We study difference schemes and having given a time-step $\Delta t$ and an uniform grid with space increment $\Delta x$, we set $\lambda=\Delta t / \Delta x$ and $x_{j}=j \Delta x, t_{n}=n \Delta t$.

The schemes we study here are based on a splitting method. We first solve the conservative system

$$
\partial_{t} U+\partial_{x} F(U)=0,
$$

and then we perform a projection onto the constraint states

$$
\partial_{t} U=Q V, \quad 1 \leq U_{1} \leq 1, \quad Q \leq 0, \quad\left(1-U_{1}\right) Q=0 .
$$

The first step of the scheme is suppose to be a flux-splitting scheme, as proposed in [8], and is written as

$$
U_{j}^{n+1 / 2}-U_{j}^{n}+\lambda\left(F_{j+1 / 2}^{n}-F_{j-1 / 2}^{n}\right)=0,
$$

with the flux

where

$$
F_{j+1 / 2}^{n}=F^{+}\left(U_{j}^{n}\right)+F^{-}\left(U_{j+1}^{n}\right),
$$

$$
F^{+}(U)+F^{-}(U)=F(U),
$$

which means the consistence of the numerical flux.

For the second step of the splitting, we just replace $U_{1}$ by $\min \left(U_{1}, 1\right)$, while $V_{i}=U_{i} / U_{1}$ are unchanged for $i=2, \cdots, p$, that is

$$
\left\{\begin{array}{l}
\left(U_{1}\right)_{j}^{n+1}=\min \left(\left(U_{1}\right)_{j}^{n+1 / 2}, 1\right), \\
\left(V_{i}\right)_{j}^{n+1}=\frac{\left(U_{i}\right)_{j}^{n+1}}{\left(U_{1}\right)_{j}^{n+1}}=\frac{\left(U_{i}\right)_{j}^{n+1 / 2}}{\left(U_{1}\right)_{j}^{n+1 / 2}}=\left(V_{i}\right)_{j}^{n+1 / 2}, \text { for } i=2, \cdots, p .
\end{array}\right.
$$

It is consistent with the system (1.4) because setting

$$
Q_{j}^{n}=\frac{\left(U_{1}\right)_{j}^{n+1}-\left(U_{1}\right)_{j}^{n+1 / 2}}{\Delta t} \leq 0,
$$

we have

$$
\begin{aligned}
\frac{\left(U_{i}\right)_{j}^{n+1}-\left(U_{i}\right)_{j}^{n+1 / 2}}{\Delta t} & =\frac{1}{\Delta t}\left(\frac{\left(U_{1}\right)_{j}^{n+1}\left(U_{i}\right)_{j}^{n+1 / 2}}{\left(U_{1}\right)_{j}^{n+1 / 2}}-\left(U_{i}\right)_{j}^{n+1 / 2}\right) \\
& =Q_{j}^{n} \frac{\left(U_{i}\right)_{j}^{n+1 / 2}}{\left(U_{1}\right)_{j}^{n+1 / 2}} \\
& =Q_{j}^{n}\left(V_{i}\right)_{j}^{n+1 / 2} .
\end{aligned}
$$


We prove now that our splitting is consistent with the entropy weak product formulation (2.7).

\subsection{Entropy compatibility}

We have the following result.

Proposition 2.2. Any classical entropy scheme given by (2.10) which is associated to (2.9) gives an entropy scheme for (2.4) with (2.7).

Proof. We assume we have a scheme which is consistent with an entropy $\eta$. That is to say there exists a numerical flux $G_{j+1 / 2}^{n}$ such that

$$
\eta\left(U_{j}^{n+1 / 2}\right)-\eta\left(U_{j}^{n}\right)+\lambda\left(G_{j+1 / 2}^{n}-G_{j-1 / 2}^{n}\right) \leq 0
$$

with

where $\Phi$ is a function such that

$$
G_{j+1 / 2}^{n}=\Phi\left(U_{j}^{n}, U_{i+j}^{n}\right)
$$

$$
\Phi(V, V)=G(V), \quad V \in \mathbb{R}^{p},
$$

where $G$ is the entropy flux associated to $\eta$.

We write

$$
\eta\left(U_{j}^{n+1}\right)-\eta\left(U_{j}^{n}\right)+\lambda\left(G_{j+1 / 2}^{n}-G_{j-1 / 2}^{n}\right) \leq \eta\left(U_{j}^{n+1}\right)-\eta\left(U_{j}^{n+1 / 2}\right) .
$$

Now if $\left(U_{1}\right)_{j}^{n+1 / 2}>1$, then $\left(U_{1}\right)_{j}^{n+1}=1$, and

$$
\eta\left(U_{j}^{n+1}\right)-\eta\left(U_{j}^{n+1 / 2}\right)=\eta\left(1, \frac{\left(U_{2}\right)_{j}^{n+1 / 2}}{\left(U_{1}\right)_{j}^{n+1 / 2}}, \cdots, \frac{\left(U_{p}\right)_{j}^{n+1 / 2}}{\left(U_{1}\right)_{j}^{n+1 / 2}}\right)-\eta\left(\left(U_{1}\right)_{j}^{n+1 / 2},\left(U_{2}\right)_{j}^{n+1 / 2}, \cdots,\left(U_{p}\right)_{j}^{n+1 / 2}\right)
$$

is a first order approximation of

$$
\begin{aligned}
& \eta^{\prime}\left(1,\left(V_{2}\right)_{j}^{n+1 / 2}, \cdots,\left(V_{p}\right)_{j}^{n+1 / 2}\right) \\
& \cdot\left(1-\left(U_{1}\right)_{j}^{n+1 / 2},\left(V_{2}\right)_{j}^{n+1 / 2}-\left(U_{2}\right)_{j}^{n+1 / 2}, \cdots,\left(V_{p}\right)_{j}^{n+1 / 2}-\left(U_{p}\right)_{j}^{n+1 / 2}\right) \\
= & \eta^{\prime}\left(1,\left(V_{2}\right)_{j}^{n+1 / 2}, \cdots,\left(V_{p}\right)_{j}^{n+1 / 2}\right) \cdot\left(1,\left(V_{2}\right)_{j}^{n+1 / 2}, \cdots,\left(V_{p}\right)_{j}^{n+1 / 2}\right)\left(1-\left(U_{1}\right)_{j}^{n+1 / 2}\right) \\
= & Q_{j}^{n} \Delta t \eta^{\prime}\left(1,\left(V_{2}\right)_{j}^{n+1 / 2}, \cdots,\left(V_{p}\right)_{j}^{n+1 / 2}\right) \cdot\left(1,\left(V_{2}\right)_{j}^{n+1 / 2}, \cdots,\left(V_{p}\right)_{j}^{n+1 / 2}\right) \cdot
\end{aligned}
$$

The inequality (2.16) is thus a first order approximation of (2.7).

We turn now to the scalar case $(p=1)$ for which the situation with a constraint is similar than without.

\subsection{Scalar case}

For a flux $F \in C^{1}(\mathbb{R}, \mathbb{R})$, we have to study

$$
\left\{\begin{array}{l}
\partial_{t} U+\partial_{x} F(U)=Q \\
0 \leq U \leq 1, \quad Q \leq 0 \\
\partial_{t}|U-k|+\partial_{x}(\operatorname{sgn}(U-k)(F(U)-F(k))) \leq Q, \quad \forall k \in[0,1]
\end{array}\right.
$$

with an initial data $U(0, x)=U^{0} \in L^{1}(\mathbb{R})$. 
We notice that we just have to consider the entropy condition for $k \in[0,1]$, instead of $k \in \mathbb{R}$, because of the constraint $0 \leq U \leq 1$.

It is well-known that the classical problem

$$
\left\{\begin{aligned}
\partial_{t} U+\partial_{x} F(U) & =0, \\
\partial_{t}|U-k|+\partial_{x}(\operatorname{sgn}(U-k)(F(U)-F(k))) & \leq 0, \quad \forall k \in \mathbb{R},
\end{aligned}\right.
$$

has an unique solution for $U^{0} \in L^{1}(\mathbb{R}) \cap L^{\infty}(\mathbb{R})$, which satisfy besides

$$
\underset{y}{\operatorname{essinf}} U^{0}(y) \leq U(t, x) \leq \underset{y}{\operatorname{esssup}} U^{0}(y), \quad \text { a.e. } x \in \mathbb{R} .
$$

Thus for an initial data $U^{0} \in L^{1}(\mathbb{R}) \cap L^{\infty}(\mathbb{R})$ such that $0 \leq U^{0} \leq 1$, the solution of (2.18) is a solution of (2.17) with $Q=0$.

Furthermore in this case, the problem with a constraint is in fact the same than without. Indeed, we have the following result, due to [18] which comes from Kružkov argument [16].

Proposition 2.3 (Lévi). There is uniqueness for the problem (2.17) in $L^{1}(\mathbb{R})$.

Remark 2.2. For a numerical treatment of the scalar case, we can use for example a classical method as Lax-Friedrichs, Engquist-Osher with a CFL condition $\lambda\left\|F^{\prime}\right\|_{L^{\infty}([0,1])} \leq 1$ in order to assure $0 \leq U_{j}^{n} \leq 1$ for the approximate solution and the convergence to the entropy solution.

\section{Euler isentropiC}

In this section, we study in more details the case of isentropic gas dynamics. In particular, we obtain a numerical scheme which is entropy consistant for the system with unilateral constraint and we prove its convergence to an entropy solution using compensated compactness.

\subsection{Theoretical result}

We first recall the main theoretical results obtain in [4]. The measure $Q$ could contain Dirac distributions in time, thus we consider weak solutions for this problem. The following existence result was proved.

Theorem 3.1 (Berthelin, Bouchut). Let $\rho^{0} \in L^{1}(\mathbb{R})$ such that $0 \leq \rho^{0} \leq 1$ and $u^{0} \in L^{\infty}(\mathbb{R})$. Then there exists $(\rho, u, Q, v)$ with regularities

$$
\begin{gathered}
\rho \in L_{t}^{\infty}\left(0, \infty ; L_{x}^{\infty}(\mathbb{R}) \cap L_{x}^{1}(\mathbb{R})\right), \\
u \in L_{t}^{\infty}\left(0, \infty ; L_{x}^{\infty}(\mathbb{R})\right), \\
Q \in \mathcal{M}\left(\left[0, \infty[\times \mathbb{R}), \quad v \in L^{\infty}(Q),\right.\right.
\end{gathered}
$$

satisfying for all $\varphi \in \mathcal{D}([0, \infty[\times \mathbb{R})$,

$$
\begin{gathered}
\int_{0}^{\infty} \int_{\mathbb{R}}\left[\rho \partial_{t} \varphi+\rho u \partial_{x} \varphi\right] \mathrm{d} t \mathrm{~d} x+\int_{\mathbb{R}} \rho^{0}(x) \varphi(0, x) \mathrm{d} x=-\int_{[0, \infty[} \int_{\mathbb{R}} \varphi Q \\
\int_{0}^{\infty} \int_{\mathbb{R}}\left[\rho u \partial_{t} \varphi+\left(\rho u^{2}+p(\rho)\right) \partial_{x} \varphi\right] \mathrm{d} t \mathrm{~d} x+\int_{\mathbb{R}} \rho^{0}(x) u^{0}(x) \varphi(0, x) \mathrm{d} x=-\int_{[0, \infty[} \int_{\mathbb{R}} \varphi Q v,
\end{gathered}
$$

and (2.2, 2.3).

Stability result of the solutions comes in particular from the following result. 
Lemma 3.2. Let $Q_{n}$ be nonpositive measures and $v_{n} \in L^{\infty}\left(Q_{n}\right)$. If $\left(Q_{n}\right)_{n \geq 0}$ is a sequence bounded in $\mathcal{M}_{\text {loc }}\left(\left[0, \infty[\times \mathbb{R})\right.\right.$ and $\left(\left\|v_{n}\right\|_{L^{\infty}\left(Q_{n}\right)}\right)_{n \geq 0}$ is bounded, then there exists a measure $Q$ and a function $v \in L^{\infty}(Q)$ such that after extraction of a subsequence, $Q_{n} \rightarrow Q, Q_{n} v_{n} \rightarrow Q v$ and $Q_{n} S\left(v_{n}\right) \rightarrow Q^{S} \leq Q S(v)$ for any convex function $S$.

We turn now to our scheme in this special case and prove its convergence to a solution of the theoretical problem.

\subsection{Flux-splitting scheme with constraint}

We have to detail more precisely the splitting method of Section 2 in this particular system. For the first step, we use a flux-splitting scheme to solve the isentropic gas dynamics, and for the second step we replace $\rho$ by $\min (\rho, 1)$, while $u$ is unchanged.

First step: We solve the isentropic system

$$
\left\{\begin{array}{l}
\partial_{t} \rho+\partial_{x}(\rho u)=0 \\
\partial_{t}(\rho u)+\partial_{x}\left(\rho u^{2}+\kappa \rho^{\gamma}\right)=0 .
\end{array}\right.
$$

We use the notation $U=(\rho, \rho u)$. The flux of the system is here $F(U)=\left(\rho u, \rho u^{2}+\kappa \rho^{\gamma}\right)$. We consider the flux-splitting scheme proposed in [8], which has the property to be entropy compatible. This scheme writes

$$
U_{j}^{n+1 / 2}-U_{j}^{n}+\lambda\left(F_{j+1 / 2}^{n}-F_{j-1 / 2}^{n}\right)=0,
$$

with the flux

$$
F_{j+1 / 2}^{n}=F^{+}\left(U_{j}^{n}\right)+F^{-}\left(U_{j+1}^{n}\right)
$$

where

$$
\begin{aligned}
& F^{+}(U)=\int_{\xi \geq 0} \xi(1,(1-\theta) u+\theta \xi)\left(a_{\gamma} \rho^{\gamma-1}-(\xi-u)^{2}\right)_{+}^{\frac{1-\theta}{2 \theta}} \mathrm{d} \xi \\
& F^{-}(U)=\int_{\xi \leq 0} \xi(1,(1-\theta) u+\theta \xi)\left(a_{\gamma} \rho^{\gamma-1}-(\xi-u)^{2}\right)_{+}^{\frac{1-\theta}{2 \theta}} \mathrm{d} \xi
\end{aligned}
$$

with $\left.a_{\gamma}=2 \sqrt{\gamma \kappa} /(\gamma-1), \gamma \in\right] 1,3\left[\right.$. We notice that $F(U)=F^{+}(U)+F^{-}(U)$. The Maxwellian which leads to this kinetic decomposition of the flux gives a BGK model which is compatible with all entropies and tends at the relaxation limit to the system of Euler isentropic with all entropies (see $[2,3,6]$ and [7]) for more details).

The scheme can be rewritten as

$$
U_{j}^{n+1 / 2}=\frac{1}{2}\left(U_{j-1 / 2}^{+}+U_{j+1 / 2}^{-}\right),
$$

with

$$
U_{j-1 / 2}^{+}=U_{j}^{n}-2 \lambda\left(F^{+}\left(U_{j}^{n}\right)-F^{+}\left(U_{j-1}^{n}\right)\right),
$$

and

$$
U_{j+1 / 2}^{-}=U_{j}^{n}-2 \lambda\left(F^{-}\left(U_{j+1}^{n}\right)-F^{-}\left(U_{j}^{n}\right)\right) .
$$

Second step: We perform a projection onto the constraint states

$$
\left\{\begin{array}{l}
\partial_{t} \rho=Q, \\
\partial_{t}(\rho u)=Q u,
\end{array} \quad 1 \leq \rho \leq 1, \quad Q \leq 0, \quad(1-\rho) Q=0 .\right.
$$


We take

and we set

$$
\left\{\begin{array}{l}
\rho_{j}^{n+1}=\min \left(\rho_{j}^{n+1 / 2}, 1\right), \\
u_{j}^{n+1}=u_{j}^{n+1 / 2}
\end{array}\right.
$$

$$
Q_{j}^{n}=\frac{\rho_{j}^{n+1}-\rho_{j}^{n+1 / 2}}{\Delta t} \leq 0 .
$$

As noted in Section 2, this splitting is consistant with the system. We prove now the convergence of this scheme to an entropy solution.

\subsection{Formulation of the scheme with Riemann problems}

For scalar conservation laws, to establish a convergence result for a scheme, we mainly use TVD and $L^{\infty}$ stability properties. For a system, the compactness of the approximated solutions of the scheme do not come from $B V$ estimates and we rather use compensated compactness techniques. The property of $L^{\infty}$ stability can be obtain using invariant domains.

We refer to [12] for the convergence to scalar equations, to $[8,9]$ for the convergence to the system of Euler and to [17] for the convergence in the case of regular systems.

One tool of our proof is the existence of global solution to the Riemann problem with arbitrary left and right states. The corresponding result we use is proved in [8], for the Riemann problem associated to the system

$$
\partial_{t} w+\partial_{x} F^{ \pm}(w)=0,
$$

with the flux $F^{ \pm}$defined by (3.9),

Theorem 3.3 (Chen, LeFloch). The Riemann problem with arbitrary left and right states for the system (3.14) admits a globally defined solution composed of two elementary waves that are either a shock wave or a rarefaction wave. Each shock wave with speed $\sigma$ connecting the left state $U_{l}$ with the right state $U_{r}$ satisfies the entropy inequality

$$
\sigma\left(\eta\left(U_{r}\right)-\eta\left(U_{l}\right)\right)-\left(G\left(U_{r}\right)-G\left(U_{l}\right)\right) \geq 0,
$$

for any convex entropy-entropy flux pair $(\eta, G)$ of the system (3.6). Furthermore, the system admits bounded and convex invariant regions for the Riemann problem.

We notice that the associated CFL condition is here

$$
\sup \left|u \pm \sqrt{\gamma \kappa} \rho^{\theta}\right| \lambda \leq \frac{1}{2} .
$$

We now define our approximated solution $U_{\Delta}$. Let $U^{0}=\left(\rho^{0}, \rho^{0} u^{0}\right)$ be an initial data such that

$$
\rho^{0} \in L^{1}(\mathbb{R}), \quad 0 \leq \rho^{0} \leq 1, \quad u^{0} \in L^{\infty}(\mathbb{R}) .
$$

We set $t_{0}=0$ and we consider the discretization of the initial data as

$$
\left.U_{\Delta}(0, x)=\frac{1}{\Delta x} \int_{x_{j-1 / 2}}^{x_{j+1 / 2}} U^{0}(y) \mathrm{d} y, \quad \text { for } x \in\right] x_{j-1 / 2}, x_{j+1 / 2}[.
$$

We assume that $U_{\Delta}(t, x)$ is defined for $t \leq t_{n}$, with $n \in \mathbb{N}$, and we define it on later time by the following way. We note $\tilde{U}_{\Delta}^{ \pm}$the global solution (on $] x_{j}, x_{j+1}[$ ) of the previous theorem for the Riemann problem (3.14) with the initial data

$$
\begin{cases}U_{j}^{n} & \text { for } x<x_{j+1 / 2}, \\ U_{j+1}^{n} & \text { for } x>x_{j+1 / 2},\end{cases}
$$


where $U_{j}^{n}$ is the averaging of $U_{\Delta}\left(t_{n}-0, x\right)$ on $] x_{j-1 / 2}, x_{j+1 / 2}[$. We set

$$
\tilde{U}_{\Delta}=\frac{1}{2}\left(\tilde{U}_{\Delta}^{+}+\tilde{U}_{\Delta}^{-}\right)
$$

on $\left\{x_{j}<x<x_{j+1}, t_{n}<t<t_{n+1}\right\}$.

We notice that by an integration of (3.14) and using

$$
F^{+}\left(\tilde{U}_{\Delta}^{+}\left(t, x_{j+1 / 2}\right)\right)=F^{+}\left(U_{j}^{n}\right), \quad F^{-}\left(\tilde{U}_{\Delta}^{-}\left(t, x_{j+1 / 2}\right)\right)=F^{-}\left(U_{j+1}^{n}\right),
$$

we have

$$
\begin{aligned}
& \frac{1}{\Delta x} \int_{x_{j-1 / 2}}^{x_{j+1 / 2}} \tilde{U}_{\Delta}\left(t_{n+1}-0, x\right) \mathrm{d} x= \\
& \frac{1}{\Delta x} \int_{x_{j-1 / 2}}^{x_{j+1 / 2}} \tilde{U}_{\Delta}\left(t_{n}-0, x\right) \mathrm{d} x-\lambda\left(F^{+}\left(U_{j}^{n}\right)-F^{+}\left(U_{j-1}^{n}\right)+F^{-}\left(U_{j+1}^{n}\right)-F^{-}\left(U_{j}^{n}\right)\right),
\end{aligned}
$$

and thus

$$
U_{j}^{n+1 / 2}=\frac{1}{\Delta x} \int_{x_{j-1 / 2}}^{x_{j+1 / 2}} \tilde{U}_{\Delta}\left(t_{n+1}-0, x\right) \mathrm{d} x .
$$

We set now

$$
U_{j}^{n+1}=\left(\rho_{j}^{n+1}, \rho_{j}^{n+1} u_{j}^{n+1}\right),
$$

with

$$
\rho_{j}^{n+1}=\min \left(1, \rho_{j}^{n+1 / 2}\right), \quad u_{j}^{n+1}=u_{j}^{n+1 / 2} .
$$

If $\rho_{j}^{n+1 / 2}>1$, we set

$$
U_{\Delta}=\frac{\tilde{U}_{\Delta}}{\rho_{j}^{n+1 / 2}}, \quad \text { on }\left\{x_{j-1 / 2}<x<x_{j+1 / 2}, t_{n}<t<t_{n+1}\right\},
$$

on the contrary, we set $U_{\Delta}=\tilde{U}_{\Delta}$.

We notice that

$$
U_{j}^{n+1}=\frac{1}{\Delta x} \int_{x_{j-1 / 2}}^{x_{j+1 / 2}} U_{\Delta}\left(t_{n+1}-0, x\right) \mathrm{d} x,
$$

and that a maximum principle holds for $U_{\Delta}$ because it holds for $\tilde{U}_{\Delta}$ from the Theorem 3.3 and (3.20) do not increase the observable values. We also have

$$
0 \leq \rho_{\Delta} \leq 1 .
$$

The sequence $\left(U_{j}^{n}\right)$ coincide with the one of the previous section. With this formulation of the scheme via Riemann problems, we prove now the convergence of the numerical method.

\subsection{Convergence of the scheme}

We have the following result.

Theorem 3.4. For initial data $\rho^{0}, u^{0}$ with regularities (3.15), there exists $U=(\rho, \rho u), Q$ and $v$ with regularities (3.1)-(3.3) such that $U_{\Delta} \rightarrow U$ a.e. and in $L_{\mathrm{loc}}^{1}(] 0,+\infty[\times \mathbb{R})$ as $\Delta \rightarrow 0$, where $(U, Q, V)$ is an entropy solution of $(3.4,3.5)$ under the constraints (2.2) and (2.3).

Remark 3.1. By $\Delta \rightarrow 0$, we mean the convergence of a sequence $\Delta_{k} x \rightarrow 0$ with $\lambda=\Delta_{k} t / \Delta_{k} x$ kept constant. Thus $U_{\Delta} \rightarrow U$ correspond to the convergence of $U_{\Delta_{k} x} \rightarrow U$ as $k \rightarrow+\infty$. 
Proof. We use the Green formula and get for any $\varphi \in \mathcal{D}\left(\left[0, t_{m}\right] \times \mathbb{R}\right)$ with $m \in \mathbb{N}^{*}$,

$$
\iint_{\left[0, t_{m}\right] \times \mathbb{R}}\left(\eta\left(U_{\Delta}\right) \partial_{t} \varphi+G\left(U_{\Delta}\right) \partial_{x} \varphi\right) \mathrm{d} x \mathrm{~d} t+\int_{\mathbb{R}} \varphi(0, x) \eta\left(U_{\Delta}(0, x)\right) \mathrm{d} x=R_{\Delta}^{\eta}(\varphi)-Q_{\Delta}^{\eta}(\varphi),
$$

where

$$
\begin{aligned}
R_{\Delta}^{\eta}(\varphi)= & \int_{0}^{t_{m}} \sum\{\sigma[\eta]-[G]\} \varphi(t, x(t)) \mathrm{d} t \\
& +\sum_{j, n} \int_{x_{j-1 / 2}}^{x_{j+1 / 2}}\left(\eta\left(U_{\Delta}\left(t_{n}-0, x\right)\right)-\eta\left(U_{j}^{n}\right)\right) \varphi\left(t_{n}, x\right) \mathrm{d} x \\
& +\int_{\mathbb{R}} \eta\left(U_{\Delta}\left(t_{m}, x\right)\right) \varphi\left(t_{m}, x\right) \mathrm{d} x-\int_{\mathbb{R}} \eta\left(U_{\Delta}(0, x)\right) \varphi(0, x) \mathrm{d} x,
\end{aligned}
$$

with the summation, for each $t>0$, over all shock waves in $U_{\Delta}(t)$ with location $x(t)$, and with

$$
Q_{\Delta}^{\eta}(\varphi)=-\sum_{n} \sum_{j ; \rho_{j}^{n+1 / 2}>1} \int_{x_{j-1 / 2}}^{x_{j+1 / 2}}\left(\eta\left(\tilde{\rho}_{\Delta}, \tilde{\rho}_{\Delta} \tilde{u}_{\Delta}\right)-\eta\left(1, \tilde{u}_{\Delta}\right)\right) \varphi\left(t_{n}, x\right) \mathrm{d} x
$$

where

For $\eta \in C^{2}$, we have

$$
\left(\tilde{\rho}_{\Delta}, \tilde{\rho}_{\Delta} \tilde{u}_{\Delta}\right)=\tilde{U}_{\Delta}
$$

$$
\eta\left(\tilde{\rho}_{\Delta}, \tilde{\rho}_{\Delta} \tilde{u}_{\Delta}\right)-\eta\left(1, \tilde{u}_{\Delta}\right)=\eta^{\prime}\left(1, \tilde{u}_{\Delta}\right) \cdot\left(1, \tilde{u}_{\Delta}\right)\left(\tilde{\rho}_{\Delta}-1\right)+\int_{0}^{1}(1-z) \eta^{\prime \prime}\left(\vartheta_{z}\right) \cdot\left(1, \tilde{u}_{\Delta}\right)^{2}\left(\tilde{\rho}_{\Delta}-1\right)^{2} \mathrm{~d} z
$$

with $\vartheta_{z}=\left[z \tilde{\rho}_{\Delta}+(1-z)\right]\left(1, \tilde{u}_{\Delta}\right)$. Now

$$
\begin{aligned}
Q_{\Delta}^{\eta}(\varphi)= & \sum_{n} \sum_{j ; \rho_{j}^{n+1 / 2}>1} \int_{x_{j-1 / 2}}^{x_{j+1 / 2}}\left[\eta^{\prime}\left(1, \tilde{u}_{\Delta}\right) \cdot\left(1, \tilde{u}_{\Delta}\right) \Delta t Q_{j}^{n}\right] \varphi\left(t_{n}, x\right) \mathrm{d} x \\
& -\sum_{n} \sum_{j ; \rho_{j}^{n+1 / 2}>1} \int_{x_{j-1 / 2}}^{x_{j+1 / 2}}\left[\int_{0}^{1}(1-z) \eta^{\prime \prime}\left(\varphi_{z}\right) \cdot\left(1, \tilde{u}_{\Delta}\right)^{2}\left(Q_{j}^{n}\right)^{2}(\Delta t)^{2} \mathrm{~d} z\right] \varphi\left(t_{n}, x\right) \mathrm{d} x,
\end{aligned}
$$

but for $\eta$ providing from a positive convex, we have (see [4])

$$
\eta^{\prime}(1, u) \cdot(1, u) \geq 0
$$

and $\eta^{\prime \prime}$ is positive definite, and thus we get that

$$
Q_{\Delta}^{\eta} \text { are nonpositive measures. }
$$

Adapting easily [8] thanks to the sign of $Q_{\Delta}^{\eta}$, we obtain

$$
R_{\Delta}^{\eta} \in \text { compact set of } H_{\mathrm{loc}}^{-1},
$$


and

$$
R_{\Delta}^{\eta} \rightarrow 0 \text { in the sense of distribution, as } \Delta \rightarrow 0,
$$

for any $C^{2}$ convex weak entropy pair $(\eta, G)$.

Using $(3.25,3.26)$ and the maximum principle on $U_{\Delta}$ (which gives $\left(\eta\left(U_{\Delta}\right)\right)_{\Delta>0}$ and $\left(G\left(U_{\Delta}\right)\right)_{\Delta>0}$ bounded in $\left.L_{t, x}^{\infty}\right)$, the sequence $\left(Q_{\Delta}^{\eta}\right)_{\Delta>0}$ is bounded in the sense of distributions, thus with (3.24) we get

$$
\left(Q_{\Delta}^{\eta}\right)_{\Delta>0} \text { is bounded in the sense of measures. }
$$

We have obtained

$$
\partial_{t} \eta\left(U_{\Delta}\right)+\partial_{x} G\left(U_{\Delta}\right)=-R_{\Delta}^{\eta}+Q_{\Delta}^{\eta}
$$

with (3.25)-(3.27), then applying the compensated compactness result of [19], we get the existence of $U \in L_{t x}^{\infty}$ such that, after extraction of a subsequence, $U_{\Delta} \rightarrow U$ a.e. and in $L_{\text {loc }}^{1}$.

We prove now the convergence of the entropy weak product. We set

$$
Q_{\Delta}=Q_{\Delta}^{\eta_{1}}, \quad \text { where } \eta_{1}(\rho, \rho u)=\rho,
$$

which is possible because $\eta_{1}$ is an entropy for the system. We have

$$
\begin{aligned}
Q_{\Delta}(\varphi) & =-\sum_{n} \sum_{j ; \rho_{j}^{n+1 / 2}>1} \int_{x_{j-1 / 2}}^{x_{j+1 / 2}}\left(\tilde{\rho}_{\Delta}-1\right) \varphi\left(t_{n}, x\right) \mathrm{d} x \\
& =\sum_{n} \sum_{j ; \rho_{j}^{n+1 / 2}>1}\left(1-\rho_{j+1 / 2}^{n}\right) \int_{x_{j-1 / 2}}^{x_{j+1 / 2}} \varphi\left(t_{n}, x\right) \mathrm{d} x,
\end{aligned}
$$

thus

$$
Q_{\Delta}=\sum_{n, j} Q_{j}^{n} \Delta t \delta_{t=t_{n}} \mathbb{1}_{x_{j-1 / 2}, x_{j+1 / 2}[}(x) \mathrm{d} x .
$$

We set now

$$
v_{\Delta}(t, x)=\sum_{n, j} u_{j}^{n+1 / 2} \mathbb{1}_{] t_{n-1 / 2}, t_{n+1 / 2}[}(t) \mathbb{1}_{]_{j-1 / 2}, x_{j+1 / 2}[}(x) .
$$

Since $v_{\Delta}$ is continuous on the Dirac measures of $Q_{\Delta}$, we can multiply these two distributions and we see that

$$
Q_{\Delta} v_{\Delta}=Q_{\Delta}^{\eta_{2}}, \quad \text { where } \eta_{2}(\rho, \rho u)=\rho u
$$

Furthermore, we have

$$
Q_{\Delta}^{\eta}=Q_{\Delta}\left[\eta^{\prime}\left(1, v_{\Delta}\right) \cdot\left(1, v_{\Delta}\right)\right]+\left(Q_{\Delta}^{\eta}\right)_{2}
$$

where

$$
\left(Q_{\Delta}^{\eta}\right)_{2}=-\sum_{n, j}\left[\int_{0}^{1}(1-z) \eta^{\prime \prime}\left(\varphi_{z}\right) \cdot\left(1, \tilde{u}_{\Delta}\right)^{2}\left(Q_{j}^{n}\right)^{2}(\Delta t)^{2} \mathrm{~d} z\right] \mathbb{1}_{x_{j-1 / 2}, x_{j+1 / 2}[} \delta_{t=t_{n}} \mathrm{~d} x \leq 0 .
$$

Now, from $(3.27)$ and $\left(U_{\Delta}\right)_{\Delta>0}$ bounded in $L_{t x}^{\infty}$, we apply Lemma 3.2 and get the existence of $Q \in \mathcal{M}_{\text {loc }}([0, \infty[\times \mathbb{R})$ and $v \in L^{\infty}(Q)$ such that, after extraction of a subsequence,

$$
\begin{aligned}
& Q_{\Delta} \rightarrow Q, \quad Q_{\Delta} v_{\Delta} \rightarrow Q v, \\
& Q_{\Delta} S\left(u_{\Delta}\right) \rightarrow Q^{S} \leq Q S(v),
\end{aligned}
$$

for any convex function $S$. We can improve this lemma in order to also get the convergence

$$
\left(Q_{\Delta}^{\eta}\right)_{2} \rightarrow \tilde{Q}^{\eta} \leq 0
$$


for any convex function $\eta$. As proved in [4], $S(v)=\eta^{\prime}(1, v) \cdot(1, v)$ is convex for $\eta$ convex, and thus

$$
Q_{\Delta}^{\eta} \rightarrow Q^{S}+\tilde{Q}^{\eta} \leq Q \eta^{\prime}(1, v) \cdot(1, v) .
$$

We conclude now the proof of the convergence of the scheme to an entropy solution of $(3.4,3.5)$ under the constraints (2.2) and (2.3) by classical arguments. We notice that the constraint $0 \leq \rho \leq 1$ comes from the similar result for $\rho_{\Delta}$.

Remark 3.2. We impose $V=U / U_{1}$, at the numerical level, nevertheless the obtained solution, at the limit $\Delta \rightarrow 0$, do not necessary satisfy such relation, which is a normal think because the theoretical problem is not a priori well posed with this relation.

\section{Equilibrium States}

In this section, we study the equilibrium states which can appear as soon as we deal with some evolution equations with a source term.

\subsection{Generalities}

Steady states are solutions in which the flux gradients are nonzero but exactly balanced by source term, that is in our class of systems,

under the constraints

$$
\partial_{x} F(U)=Q \cdot V
$$

$$
0 \leq U_{1} \leq 1, \quad Q \leq 0, \quad\left(1-U_{1}\right) Q=0,
$$

and the entropy weak product inequalities

$$
\partial_{x} G_{S}(U) \leq Q \eta^{\prime}\left(1, V_{2}, \cdots, V_{p}\right) \cdot\left(1, V_{2}, \cdots, V_{p}\right),
$$

for a sufficient family of entropies associated to the system (2.4). When using a splitting method, the scheme may break this equilibrium and does not necessary preserve it. Sometimes, it needs a high level of approximation and a high cost to get it. Besides, these equilibrium solutions play an important part because usually when time tends to infinity, general solutions to (1.4)-(1.6) might tend to (4.1)-(4.3). This notion is thus important and the subject of many works for evolution equations.

A way to study such problems is the well-balanced schemes, which reformulate the source term in order to write it as a numerical flux (see $[13,14]$ ). This technique maintains all steady states. But using exact Riemann solvers as Godunov scheme, the calculation cost is important.

An important example for the study of steady states is the Saint-Venant equation with a bottom. A kinetic study with reflexions is done in [5] which maintain the steady states and establish the convergence of the scheme. A different approach is perform in [15], adapting the value of the source term at the interface rather than its mean on the cellular.

We study in the next section the steady states for the isentropic system with constraint.

\subsection{Steady states for Euler isentropic with constraint}

For the system (1.1), the steady states are solutions to

$$
\begin{gathered}
\partial_{x}(\rho u)=Q, \\
\partial_{x}\left(\rho u^{2}+\kappa \rho^{\gamma}\right)=Q v,
\end{gathered}
$$

with the constraints

$$
0 \leq \rho \leq 1, \quad Q \leq 0, \quad(1-\rho) Q=0,
$$


and the weak entropy product

$$
\partial_{x} G_{w \mapsto w^{2} / 2}(\rho, u) \leq Q \eta_{w \mapsto w^{2} / 2}^{\prime}(1, v) \cdot(1, v)
$$

Indeed, we only have to ask the weak product formulation for $S_{1}(w)=w^{2} / 2$ in this case thanks to Remark 2.1 .

In particular the classical solutions (we mean without constraints) can satisfy it, for example

$$
\rho=\text { constant } \in[0,1], \quad u=\text { constant. }
$$

But there is also steady states with a nontrivial constraint, for example:

$$
\rho=1, \quad u=\mathbb{1}_{x<0}-\mathbb{1}_{x>0}, \quad Q=-2 \delta_{0}, \quad v=0 .
$$

These functions satisfy

$$
\begin{gathered}
(1-\rho) Q=0 \\
\partial_{x} G_{S_{1}}(\rho, u)=-2 \delta_{0}\left(\frac{1}{2}+\kappa \frac{\gamma}{\gamma-1}\right), \\
\eta_{S_{1}}^{\prime}(1, v) \cdot(1, v)=\kappa \frac{\gamma}{\gamma-1},
\end{gathered}
$$

and thus (4.7) is satisfy.

In fact, we could not preserve such steady states on a numerical point of view because Dirac mass can only been approximated. The following result states that there is not non-trivial steady states in $L^{1}$.

Proposition 4.1. Let $I=] a, b[$ be an interval of $\mathbb{R}$. Let $(\rho, u, v, Q)$ be a solution to (4.4)-(4.7) such that $\rho=1$ on $I, u, v \in L^{\infty}(I)$ and $Q \in L^{1}(I)$. Then $Q u=Q v=0$ on $I$.

Proof. We compute

$$
\eta_{w \mapsto w^{2} / 2}^{\prime}(1, v) \cdot(1, v)=\frac{v^{2}}{2}+\frac{\kappa \gamma}{\gamma-1} \text { on } I
$$

and

$$
G_{w \mapsto w^{2} / 2}(\rho, u)=\frac{1}{2} u^{3}+\frac{\kappa \gamma}{\gamma-1} u \text { on } I .
$$

Now, from (4.4), we have $\partial_{x} u=Q$ on $I$, therefore $u \in W^{1,1}(I)$. As besides $u^{2} \in L^{\infty}(I)$, we have

$$
\begin{aligned}
\partial_{x}\left(\frac{1}{2} u^{3}+\frac{\kappa \gamma}{\gamma-1} u\right) & =\left(\frac{3}{2} u^{2}+\frac{\kappa \gamma}{\gamma-1}\right) \partial_{x} u \text { on } I \\
& =\left(\frac{3}{2} u^{2}+\frac{\kappa \gamma}{\gamma-1}\right) Q \text { on } I .
\end{aligned}
$$

Thus (4.7) leads to

which is the same than

$$
\left(\frac{3}{2} u^{2}+\frac{\kappa \gamma}{\gamma-1}\right) Q \leq\left(\frac{1}{2} v^{2}+\frac{\kappa \gamma}{\gamma-1}\right) Q \text { on } I
$$

$$
3 u^{2} Q \leq v^{2} Q \text { on } I \text {. }
$$

Now, from (4.5), we have $\partial_{x} u^{2}=Q v$ on $I$, therefore $2 u Q=Q v$ on $I$, thus using (4.8) we obtain

$$
0 \leq u^{2} Q \text { on } I
$$

and since $Q \leq 0$, we get $Q u=0$ on $I$ and also $Q v=0$.

Remark 4.1. We reduce our study to $\rho=1$ because $Q$ have to act only on this set. 


\section{Full System of Gas DYNAMics}

We study now the overflow of a river modelled by the system of full-gas dynamics. It involves the density $\rho$, the velocity $u$ and the energy $e$ of the fluid and can be written as

$$
\left\{\begin{array}{l}
\partial_{t} \rho+\partial_{x}(\rho u)=Q, \\
\partial_{t}(\rho u)+\partial_{x}\left(\rho u^{2}+p\right)=Q u, \\
\partial_{t}(\rho e)+\partial_{x}((\rho e+p) u)=Q e
\end{array}\right.
$$

where $\rho e=\rho \frac{u^{2}}{2}+\frac{1}{\gamma-1} p$, with $\gamma>1$, with constraint and mass loss rate Lagrange multiplier

$$
0 \leq \rho \leq 1, \quad p \geq 0, \quad Q \leq 0
$$

and extremality relation

$$
(1-\rho) Q=0 \text {. }
$$

Here the conservative variables are $U=(\rho, q=\rho u, E=\rho e)$. We have here a supplementary constraint which is the fact that the pression $p$ has to stay nonnegative. The entropies for this system are given for any function $S$ by

$$
\eta_{S}(\rho, q, E)=\rho S\left(\frac{p^{1 / \gamma}}{\rho}\right)
$$

These functions are convex with respect to $(\rho, q, E)$ for $S$ of class $C^{1}$ convex and nonincreasing.

With the variables $w=\frac{p^{1 / \gamma}}{\rho}$ and $\sigma=p^{1-1 / \gamma}$, we have $\eta_{S}(\rho, q, E)=\rho S(\omega)$, and

$$
\partial_{\rho} \omega=-\frac{\omega}{\rho}+\frac{1}{\sigma} \frac{1}{\rho} \frac{\gamma-1}{\gamma} \frac{u^{2}}{2}, \quad \partial_{q} \omega=-\frac{\gamma-1}{\gamma} \frac{u}{\sigma \rho}, \quad \partial_{E} \omega=\frac{\gamma-1}{\gamma} \frac{1}{\sigma \rho}
$$

thus we get

$$
\eta_{S}^{\prime}(\rho, e, u)=\left(S(\omega)+\left(-\omega+\frac{1}{\sigma} \frac{\gamma-1}{\gamma} \frac{u^{2}}{2}\right) S^{\prime}(\omega),-\frac{1}{\sigma} \frac{\gamma-1}{\gamma} u S^{\prime}(\omega), \frac{1}{\sigma} \frac{\gamma-1}{\gamma} S^{\prime}(\omega)\right) .
$$

A weak formulation which allows to deal with the products $Q \rho, Q u$ and $Q e$ is the following one:

$$
\left\{\begin{array}{l}
\partial_{t} \rho+\partial_{x}(\rho u)=Q, \\
\partial_{t}(\rho u)+\partial_{x}\left(\rho u^{2}+p\right)=Q \tilde{u}, \\
\partial_{t}(\rho e)+\partial_{x}((\rho e+p) u)=Q \tilde{e}
\end{array}\right.
$$

with as before

$$
0 \leq \rho \leq 1, \quad p \geq 0, \quad Q \leq 0 .
$$

We take $\tilde{u}, \tilde{e} \in L^{\infty}(Q)$, so that the products are well-defined. The vector $V$ related to the lost quantities is here $(1, \tilde{u}, \tilde{e})$. We also ask that we can write $\rho \tilde{e}=\rho \frac{\tilde{u} 2}{2}+\frac{1}{\gamma-1} \tilde{p}$ with $\tilde{p} \geq 0$.

We formulate in a weak sense that $Q \rho=Q, Q \tilde{u}=Q u, Q \tilde{e}=Q e$ using the procedure proposed in Section 2. Thus we require the family of entropy weak product inequalities

$$
\partial_{t} \eta_{S}(\rho, u, e)+\partial_{x} G_{S}(\rho, u, e) \leq Q \eta_{S}^{\prime}(1, \tilde{u}, \tilde{e}) \cdot(1, \tilde{u}, \tilde{e})
$$


which are here

$$
\partial_{t} \eta_{S}(\rho, u, e)+\partial_{x} G_{S}(\rho, u, e) \leq Q\left[S\left(\tilde{p}^{1 / \gamma}\right)+S^{\prime}\left(\tilde{p}^{1 / \gamma}\right)\left(\frac{1-\gamma}{\gamma} \tilde{p}^{1 / \gamma}\right)\right],
$$

for any convex entropy $\eta_{S}$ in a suitable family parametrized by a convex function $S$.

In order to see that (5.5)-(5.7) is a weak formulation of (5.1)-(5.3), we observe first that any suitable solution to (5.1)-(5.3) also solves (5.5)-(5.7) with $\tilde{u}=u$ and $\tilde{e}=e$. Conversely, if we have a sufficiently smooth solution to (5.5)-(5.7) such that $\rho>0$, then multiplying (5.5) by $\eta_{S}^{\prime}(\rho, u, e)$ and comparing the result with (5.7), we get

$Q\left[S\left(\frac{p^{1 / \gamma}}{\rho}\right)+S^{\prime}\left(\frac{p^{1 / \gamma}}{\rho}\right)\left(\frac{p^{1 / \gamma}}{\rho}\left(-1+\frac{\tilde{p}}{\gamma p}\right)+\left(1-\frac{1}{\gamma}\right) \frac{1}{2 \sigma}(u-\tilde{u})^{2}\right)\right]$

$$
\leq Q\left[S\left(\tilde{p}^{1 / \gamma}\right)+S^{\prime}\left(\tilde{p}^{1 / \gamma}\right)\left(\frac{1-\gamma}{\gamma} \tilde{p}^{1 / \gamma}\right)\right] .
$$

We use the entropy associated with $S(v)=-\ln v$ and get

$$
0 \leq Q\left[\ln \left(\frac{p^{1 / \gamma}}{\rho \tilde{p}^{1 / \gamma}}\right)-\frac{1}{\gamma}+\frac{\tilde{p}}{\gamma p}+\frac{\rho}{2 p}\left(1-\frac{1}{\gamma}\right)(u-\tilde{u})^{2}\right]
$$

which can be written as

$$
0 \leq Q\left[\frac{1}{\gamma} \Gamma\left(\frac{p}{\tilde{p}}\right)-\ln \rho+\frac{\rho}{2 p}\left(1-\frac{1}{\gamma}\right)(u-\tilde{u})^{2}\right],
$$

with $\Gamma(x)=\ln x-1+1 / x$. Now $\left(1-\frac{1}{\gamma}\right)>0$ since $\gamma>1,-\ln \rho \geq 0$ since $0<\rho \leq 1$, and $\Gamma(x) \geq 0$ for $x>0$, therefore we get that

and thus

$$
Q \Gamma\left(\frac{p}{\tilde{p}}\right)=0, \quad Q \ln \rho=0, \quad Q(u-\tilde{u})^{2}=0,
$$

$$
Q p=Q \tilde{p}, \quad Q \rho=Q, \quad Q u=Q \tilde{u} .
$$

As in the isentropic case, we see in particular that only one entropy is required to have a weak formulation. Here it is the entropy associated with $S(v)=-\ln v$.

The theoretical study of the problem without a constraint is not possible because there is no invariant domain (see [6]), thus the problem with constraint is similarly difficult. Nevertheless, we can perform numerical simulations in preserving the non-negativity of $\rho$ and $p$.

Acknowledgements. This research was supported by the French-Austrian "Amadeus" programme (ÖAD V6) and by the Austrian START project (TEC-Y137) "Nonlinear Schrödinger and quantum Boltzmann equations".

\section{REFERENCES}

[1] F. Berthelin, Existence and weak stability for a two-phase model with unilateral constraint. Math. Models Methods Appl. Sci. 12 (2002) 249-272.

[2] F. Berthelin and F. Bouchut, Solution with finite energy to a BGK system relaxing to isentropic gas dynamics. Ann. Fac. Sci. Toulouse Math. 9 (2000) 605-630.

[3] F. Berthelin and F. Bouchut, Kinetic invariant domains and relaxation limit from a BGK model to isentropic gas dynamics. Asymptot. Anal. 31 (2002) 153-176.

[4] F. Berthelin and F. Bouchut, Weak solutions for a hyperbolic system with unilateral constraint and mass loss. Ann. Inst. H. Poincaré Anal. Non Linéaire (to appear).

[5] R. Botchorishvili, B. Perthame and A. Vasseur, Equilibrium schemes for scalar conservation laws with stiff sources. Rapport INRIA RR-3891.

[6] F. Bouchut, Construction of BGK models with a family of kinetic entropies for a given system of conservation laws. J. Statist. Phys. 95 (1999) 113-170.

[7] F. Bouchut, Entropy satisfying flux vector splittings and kinetic BGK models. Numer. Math. (to appear). 
[8] G.-Q. Chen and P.G. LeFloch, Entropy flux-splittings for hyperbolic conservation laws I, General framework. Comm. Pure Appl. Math. 48 (1995) 691-729.

[9] G.-Q. Chen and P.G. LeFloch, Entropies and flux-splittings for the isentropic Euler equations. Chinese Ann. Math. Ser. B 22 (2001) 145-158.

[10] B. Després, Equality or convex inequality constraints and hyperbolic systems of conservation laws with entropy. Preprint (2001).

[11] E. Weinan, Y.G. Rykov and Y.G. Sinai, Generalized variational principles, global weak solutions and behavior with random initial data for systems of conservation laws arising in adhesion particle dynamics. Comm. Math. Phys. 177 (1996) 349-380.

[12] E. Godlewski and P.-A. Raviart, Hyperbolic systems of conservation laws. Mathématiques \& Applications 3/4, Ellipses, Paris (1991).

[13] L. Gosse and A.-Y. Le Roux, A well-balanced scheme designed for inhomogeneous scalar conservation laws. $C$. $R$. Acad. Sci. Paris Sér. I Math. 323 (1996) 543-546.

[14] J.M. Greenberg and A.-Y. Le Roux, A well-balanced scheme for the numerical processing of source terms in hyperbolic equations. SIAM J. Numer. Anal. 33 (1996) 1-16.

[15] S. Jin, A steady-state capturing method for hyperbolic systems with geometrical source term. ESAIM: M2AN 35 (2001) 631-645.

[16] S.N. Kružkov, First order quasilinear equations in several independant variables. Mat. Sb. 81 (1970) 285-255; Mat. Sb 10 (1970) 217-243.

[17] C. Lattanzio and D. Serre, Convergence of a relaxation scheme for hyperbolic systems of conservation laws. Numer. Math. $8 \mathbf{8}$ (2001) 121-134.

[18] L. Lévi, Obstacle problems for scalar conservation laws. ESAIM: M2AN 35 (2001) 575-593.

[19] P.-L. Lions, B. Perthame and P.E. Souganidis, Existence and stability of entropy solutions for the hyperbolic systems of isentropic gas dynamics in Eulerian and Lagrangian coordinates. Comm. Pure Appl. Math. 49 (1996) 599-638.

[20] F. Mignot and J.-P. Puel, Inéquations variationnelles et quasivariationnelles hyperboliques du premier ordre. J. Math. Pures Appl. 55 (1976) 353-378.

To access this journal online:

www.edpsciences.org 